\title{
Osteoporosis Targeting, Possible Breakthroughs
}

\section{Da-Yong Lu* and Jin-Yu Che}

School of Life Sciences, Shanghai University, Shanghai, China

*Corresponding Author: Da-Yong Lu, School of Life Sciences, Shanghai University, Shanghai, China.
Received: August 25, 2021

Published: October 01, 2021

(C) All rights are reserved by Da-Yong Lu and Jin-Yu Che.

\section{Abstract}

Osteoporosis is an issue of public health concern worldwide. Currently, it is very difficult in the clinic. To face with this difficulty, the therapeutic breakthrough is a top priority. This editorial aims to discuss this hot topic of orthopedic disease.

Keywords: Osteoporosis; Clinical Trial; Personalized Medicine; Orthopedic Disease

\section{Introduction}

Osteoporosis is an issue of public health concern worldwide [1-3]. Osteoporosis-induced complications have high incidence of human mortality in old people. Many clinical interventions are commonly utilized in the clinic [4-8]. However, the outcomes were unsatisfactory. To face with this difficulty, the therapeutic breakthrough is a top priority. This editorial aims to discuss this hot topic of orthopedic disease.

Available therapeutics

Available therapeutics is classified as [2]:

- Calcium pills

- Hormone therapy

- Calcitonin

- Food fortification

- Different types of drugs [4-8].

\section{Therapeutic breakthroughs}

In the clinic, the osteoporosis rate and evidence were postponed in modern society. However, these ranges of bioinformatics were not translated into modern medicine (knowledge, technology and therapies). If we can strengthen our knowledge about clinical symptom relieving or reversal [9-22] and drug development [2326], therapeutic breakthroughs can be foreseeable.

\section{Future Direction}

In the future, widely used therapeutic strategies for other deadly diseases can be available in the scope of osteoporosis treatment in the clinic [28-34]. To facilitate these researches, therapeutic breakthroughs are indispensable.

\section{Bibliography}

1. Melton J. "Hip fracture; a worldwide problem today and tomorrow". Bone 14 (1993): S1-8.

2. Lu DY and Che JY. "Osteoporosis treatments". Clinical Biotechnology and Macrobiology 3.2 (2019): 612-614.

3. Lu DY., et al. "Osteoporosis in old women, therapeutic selection”. EC Orthopaedics 9.7 (2018): 386. 
4. Choudhary D and Alam A. "Anti-osteoporotic activity of bioactive compounds from Iris germanica targeting NK-Kappa B". EC Pharmacology and Toxicology 6.8 (2018): 665-678.

5. Marks R. "Vitamin E and osteoarthritic cartilage: Does vitamin E influence cartilage integrity?” EC Orthopaedics 10.5 (2019): 281-294.

6. Patel S. "Conservative pain management". EC Orthopaedics 9.8 (2018): 621-623.

7. Lu DY., et al. "Osteoporosis, importance for early diagnosis and treatment”. EC Orthopaedics 9.9 (2018): 624-625.

8. Lu DY., et al. "Osteoporosis treatments for old people". EC Orthopeadicis 10.5 (2019): 278-280.

9. Lu DY., et al. "Bone disease recovery strategies, An overview". EC Orthopaedics 10.1 (2019): 1-3.

10. Araujo JL. "The role of the orthopedic surgeon in preventing low back pain chronification". EC Orthopaedics 9.12 (2018): 809-812.

11. Harsini SM and Oryan A. "Bone grafting and the materials for using in orthopaedics”. EC Orthopaedics 9.12 (2018): 822-833.

12. Zweedijk R., et al. "Scoliosis and osteopathy". Acta Scientific Orthopaedics 3.9 (2020): 30-43.

13. Lu DY and Che JY. "Bone disease treatment, an editorial". EC Orthopeadics 11.8 (2020): 143-145.

14. Lu DY., et al. "Bone disease recovery strategies, An overview". EC Orthopaedics 10.1 (2019): 1-3.

15. Koleva IB and Yoshinov B. "Rehabilitation as an essential element in the clinical practice of orthopaedics and traumatology". Acta Scientific Orthopaedics 3.9 (2020): 44-46.

16. Lu DY., et al. "Osteoporosis treatments for old people”. EC Orthopeadicis 10.5 (2019): 278-280.

17. Che JY and Lu DY. "Bone disease treatment, future direction". EC Pharmacology and Toxicology 9.1 (2021): 7-8.

18. Negm SH. "The possible protective role of powder cuttlefish bone, crab shell and eggshell on osteoporotic rats". Journal of Food and Dairy Sciences 9.10 (2018): 111-121.
19. Mobasheri A and Shakbael M. "Osteogenic effects of resveratrol in vitro: potential for the prevention and treatment of osteoporosis". Annals of the New York Academy of Sciences 1290 (2013): 59-66.

20. Madrv H., et al. "Scaffold-mediated gene delivery for osteochondral repair". Pharmaceutics 12 (2020): 930.

21. Lu DY and Che JY. "Bone disease treatments, technical advances". EC Orthopeadics 11.10 (2020): 1-3.

22. Lu DY., et al. "Bone disease treatments, math-therapeutic modality”. EC Orthopaedics 10.3 (2019): 140-143.

23. Lu DY and Lu TR. "Drug discoveries from natural resources". Journal of Primary Care and General Practice 3.1 (2019): 28.

24. Muthuraman A., et al. "Integration of artificial intelligence in pharmacological research with deep and machine learning process". EC Pharmacology and Toxicology 7.11 (2019): 56-61.

25. Freedman DH. "Hunting for new drugs with AI". Nature 576 (2019): s49-s53.

26. Borrego-Gonzalez S., et al. "Sponge-like processed D-periodic self-assembled atelocollagen supports bone formation in vivo". Materials Science and Engineering C (2020).

27. Leung PC. "Traditional Chinese medicine in orthopaedicsproblems and future direction". Open Journal of Therapy and Rehabilitation 2.1 (2014): 1-4.

28. Lu DY. "Personalized cancer chemotherapy, an effective way for enhancing outcomes in clinics. 2014, Woodhead Publishing, Elsevier, UK (2014).

29. Lu DY., et al. "Individualized cancer therapy, future approaches". Current Pharmacogenomics and Personalized Medicine 16.2 (2018): 156-163.

30. Lu DY., et al. "HAART in HIV/AIDS treatments, future trends". Infectious Disorders-Drug Targets 18.1 (2018): 15-22.

31. Lu DY., et al. "HIV/AIDS curable study, new forms of therapeutic trinity". Recent Patents on Anti-Infective Drug Discovery 13.3 (2018): 217-227. 
32. Lu DY., et al. "Anticancer drug combination, how far we can go through?" Anti-Cancer Agents in Medicinal Chemistry 17.1 (2017): 21-28.

33. Lu DY., et al. "Drug combination in clinical cancer treatment". Reviews on Recent Clinical Trials 12.3 (2017): 202-211.

34. Lu DY., et al. "Individualized cancer therapy, what is the next generation?" EC Cancer 2.6 (2018): 286-297.

Volume 4 Issue 11 November 2021

(C) All rights are reserved by Da-Yong Lu and Jin-Yu Che. 